\title{
Beam Footprint Detection and Tracking for Non-Cooperative Bistatic SAR
}

\author{
Feifei YAN, Wenge CHANG, Xiangyang LI, Qilei ZHANG \\ College of Electronic Science and Engineering, National University of Defense Technology, \\ Changsha, Hunan, P. R. China, 410073 \\ ddx_yff@163.com, changwenge@ nudt.edu.cn, lxyniu@sina.com, zhangqilei1985@gmail.com
}

Manuscript received March 11, 2016

\begin{abstract}
In non-cooperative bistatic synthetic aperture radar (SAR), the position of transmitter's beam footprint should be detected and tracked in real-time to perform beam synchronization. Theoretical analysis shows that signal-tonoise ratio (SNR) of the reflected echoes from the observational scene is too low to apply the conventional detection and tracking method. According to the cross-correlation and Doppler frequency information of the backscattering echo, a beam footprint detection and tracking method is proposed in this paper. This method can realize accumulation of signal energy, therefore enormously improve the performance of beam footprint detection and tracking. Meanwhile, vehiclebased bistatic SAR experiment and airborne bistatic SAR experiment are performed to evaluate the performance of the proposed beam footprint detection and tracking method. Experimental results show that the proposed method performs well for real-time transmitter beam footprint detection and tracking.
\end{abstract}

\section{Keywords}

Beam footprint, detection and tracking, noncooperative, cross-correlation, Doppler information

\section{Introduction}

Non-cooperative bistatic SAR, which uses spaceborne or airborne radar as the opportunity illuminator, has recently been recognized as an interesting research hotspot [1], [2]. Non-cooperative radars, despite its low system cost, can also achieve other benefits like improved feature extraction and classification of targets, increased surveillance for applications in conflict areas. However, bistatic SAR uses separate antennas for transmission and reception, this spatial separation poses synchronization challenges between the transmitter and the receiver. One of the synchronization challenges is the transmitter's real-time beam footprint detection and tracking. For the case of non-cooperative transmitter, as there is no special synchronization link between the transmitter and the receiver, the accurate arrival time and position of the trans- mitter's beam footprint cannot be known in real-time, but the anticipative observational area can be forecasted. So the sampling signal from the anticipative observed area should be analyzed in real-time to detect whether the transmitter's beam footprint is coming. After that, the synchronization mechanism of the receiver can realize beam synchronization.

To realize beam synchronization in non-cooperative bistatic SAR, there are two important problems to solve. One of which is the real-time detection for the arrival time of the transmitter's beam footprint [3-5]; the other is the computation of the relative position between the transmitter and the receiver [6], [7]. As the anticipative observational area can be forecasted, so the main work of the beam synchronization for non-cooperative bistatic SAR is to realize transmitter's beam detection and tracking in the azimuth direction. As the reflected echo from the observational area is a weak linear frequency modulation (LFM) signal, so the first problem can be seen as weak signal detection. There are some classical methods for weak signal detection. Traditional methods include fast Fourier transform (FFT), filtering, and lock-in amplifier, etc. As the SNR of reflected echoes is very low, the detection performance is poor. The stochastic resonance and wavelets are suitable to detect the LFM signals because of its unique properties [8], [9]. However, the computational time is very long, and thus the weak signal detection cannot be realized in real-time. In summary, few studies have been reported about beam footprint detection for non-cooperative spaceborne/airborne bistatic SAR.

For the second problem, a double sliding spotlight mode is proposed in [10]. By reducing the footprint's velocity difference between the transmitter and the receiver, it can achieve a tradeoff between the azimuth resolution and the scene extension. But some assumptions significantly limit its practicability in non-cooperative bistatic SAR system. The comparison of attitude and antenna pointing design strategies for non-cooperative spaceborne bistatic radar were investigated in [11]. Two different beam synchronization methods are reported there. However, these methods do not include any beam detection mechanisms. Consequently, although the beam footprint synchronization methods have already been demonstrated, transmitter's beam footprint detection 


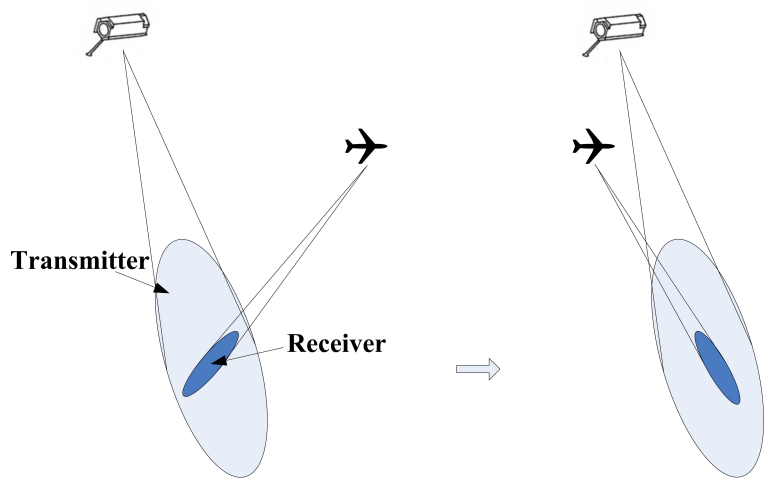

Fig. 1. Relative motion of beam footprint.

and tracking, especially the real-time realization of them, is still a technical challenge in non-cooperative bistatic SAR. Few studies have been reported for this topic.

In this paper, an efficient transmitter's beam detection and tracking method for non-cooperative bistatic SAR is proposed. Based on cross-correlation between the echoes of adjacent pulse repetition frequency (PRF) and $\mathrm{M} / \mathrm{N}$ detection, the efficiency of transmitter's footprint detection is greatly improved. Meanwhile, according to the Doppler spectrum of the accumulated signal, the relative position between the transmitter and the receiver can be obtained. To better analyze the performance of the proposed method, vehicle-based bistatic SAR experiment and airborne bistatic SAR experiment are performed. The remainder of the paper is arranged as follows: Section 2 introduces the beam detection and tracking method. Section 3 briefly introduces of the vehicle-based bistatic SAR experiment and the airborne bistatic SAR experiment. The results of the two experiments are presented in Section 3 to verify the proposed method. Finally, some conclusions are drawn in Section 4.

\section{Beam Footprint Detection and Tracking}

Figure 1 shows the beam footprints of the transmitter and the receiver, respectively. Suppose the transmitter is working in stripmap mode. The velocity of satellite is far quicker than the airplane's, and theoretical analysis shows that the beam width of the receiver is far less than those of the transmitter in spaceborne/airborne bistatic SAR system. If the receiver is also working in stripmap mode, the scene extension is quite limited. To achieve a tradeoff between the azimuth resolution and the scene extension, beam footprint detection and tracking should be implemented in non-cooperative bistatic SAR.

In non-cooperative bistatic SAR system, the ephemeris and the observational area of the transmitter can be acquired in advance, so the receiver's beam footprint can aim at the given observational area for a period of time without any synchronization. Due to the high velocity difference between the transmitter and the receiver, the receiver's beam footprint can be considered to be fixed. When the overlap of the beam footprint between the transmitter and the receiver is gradually increased, the cross-correlation result becomes larger. When the cross-correlation result exceeds the threshold, the Doppler spectrum of the accumulated signal is analyzed to get the beam footprint center distance between the transmitter and the receiver. If the beam footprint center distance satisfies the predefined condition [12], according to the velocity and direction of the transmitter's beam footprint, the receiver's beam footprint moves to pursue the transmitter's beam footprint to realize transmitter's beam synchronization.

\subsection{Beam Footprint Detection}

Since the observational scenes of the adjacent PRF are almost the same, the adjacent sampling echoes have high correlation [3]. As the noises in the adjacent PRFs are independent, the transmitter's beam footprint detection can be realized by cross-correlation approach. To improve the detection performance, the correlation result is cut in the time domain to reduce the noise. Then FFT and noncoherent integration are performed for $\mathrm{M} / \mathrm{N}$ detection. The proposed detection method can realize the accumulation of the signal energy without range correction migration correct, so the performance of the proposed beam footprint detection method can be enormously improved.

\section{(1) Cross-correlation}

The sampling echoes of the adjacent PRF can be written as:

$$
\left\{\begin{array}{l}
x_{1}(\tau, \eta)=s_{r}(\tau, \eta)+\omega_{1}, \\
x_{2}(\tau, \eta)=s_{r}(\tau, \eta+T)+\omega_{2}
\end{array}\right.
$$

where $T=1 / \mathrm{PRF}, s_{r}(\tau, \eta)$ and $s_{r}(\tau, \eta+T)$ are the adjacent sampling echoes, respectively. $\omega_{1}$ and $\omega_{2}$ are white Gaussian noises of the adjacent PRF with zero mean and standard deviation $\sigma_{2}$, respectively.

The cross-correlation result of the adjacent sampling echoes is:

$$
\begin{aligned}
R_{x_{1} x_{2}} & =R_{s}+R_{s \omega_{1}}+R_{s \omega_{2}}+R_{\omega_{1} \omega_{2}} \\
& =R_{s}+R_{\omega}
\end{aligned}
$$

where $R_{\omega}$ is:

$$
\begin{aligned}
R_{\omega} & =R_{s \omega_{1}}+R_{s \omega_{2}}+R_{\omega_{1} \omega_{2}}, \\
& =\frac{1}{N} \Sigma\left[s_{r}^{*}(\tau, \eta+T) \omega_{1}+s_{r}(\tau, \eta) \omega_{2}^{*}+\omega_{1} \omega_{2}^{*}\right] .
\end{aligned}
$$

When $N$ is large enough, according to the central limit theorems in probability theory [13], $R_{\omega}$ is nearly white Gaussian noise with zero mean and standard deviation $\left(2 E_{s} \sigma_{2}+\sigma_{4}\right) / N . E_{s}$ is the energy summation of the backscattering signal. When the sampling echo is only noise, the real and imaginary part of $R_{x_{1} x_{2}}(\tau)$ are independent, which are white Gaussian noise with zero mean and standard deviation $\sigma_{4} / N$. The PDF of $R_{x_{1} x_{2}}(\tau)$ is: 


$$
P(x)= \begin{cases}\frac{x}{\sigma_{4} / N} \exp \left(\frac{x}{2 \cdot\left(\sigma_{4} / N\right)}\right) & \text { for } \quad 0 \leq x<\infty, \\ 0 & \text { for } \quad x<0 .\end{cases}
$$

In CFAR systems [14], the probability of false alarm is:

$$
\begin{aligned}
P_{\mathrm{FA}} & =\int_{V_{T}}^{\infty}\left(X>T Z \mid H_{0}\right) f_{z}(z) \mathrm{d} x, \\
& =E_{z}\left(P\left(X>T Z \mid H_{0}\right)\right)
\end{aligned}
$$

where $Z$ is the mean clutter power, $T$ is a scaling factor and $f_{z}(z)$ is the probability density function of $Z$.

Substituting $f_{z}(z)$ into (5), $P_{\mathrm{FA}}$ is:

$$
P_{\mathrm{FA}}=M_{z}\left(\frac{T}{2 \sigma_{2}}\right)
$$

where $M_{z}(\cdot)$ is the moment-generating function of $Z$. So if the false alarm probability $P_{\mathrm{FA}}$ is given, we can get the threshold $V_{T}$.

\section{(2) $\mathrm{M} / \mathrm{N}$ detection}

The cross-correlation result of the adjacent sampling echoes cannot determine whether the received signal is LFM signal arisen from the transmitter radar. As the signal bandwidth of the transmitter can be obtained from the direct path signal, then $\mathrm{M} / \mathrm{N}$ detection is performed after noncoherent integration to distinguish the sampling signal between the transmitter's LFM signal and other signal in frequency domain [15].

The M/N detection can be written as:

$$
\Sigma_{i=1}^{N} u\left(z_{i}-T\right) \gtrless_{H_{0}}^{H_{1}}, \quad u(z)=1 \quad \text { or } \quad 0
$$

where $N$ is the sampling point in the range dimension.

Let $P_{\mathrm{d}, \text { cell }}$ denote the probability that any given pulse exceeds the threshold. Let $P_{\mathrm{d}}$ be the probability that the signal is detected, i.e., the threshold is crossed $M_{p}$ times within $N_{p}$ looks. Then, from (7), we derive:

$$
P_{\mathrm{d}}=\Sigma_{m=M_{p}}^{N_{p}} \frac{N_{p} !}{m !\left(N_{p}-M_{p}\right) !}\left(P_{\mathrm{d}, \mathrm{cell}}\right)^{m}\left(1-P_{\mathrm{d}, \mathrm{cell}}\right)^{N_{p}-m} .
$$

The double threshold probability of false alarm is:

$$
P_{\mathrm{FA}}=\Sigma_{m=M_{p}}^{N_{p}} \frac{N_{p} !}{m !\left(N_{p}-M_{p}\right) !}\left(P_{\mathrm{f}, \text { cell }}\right)^{m}\left(1-P_{\mathrm{f}, \text { cell }}\right)^{N_{p}-m} .
$$

As can be seen from (4), after cross-correlation, $\omega$ is a white Gaussian noise with zero mean and standard deviation $\sigma_{4} / N$. When only noise is present, the standard deviation of the noise after FFT is determined by the width $W$ of the rectangle window. Then the real and imaginary parts of the noise are white Gaussian noise with zero mean and standard deviation $2 W \sigma_{p}^{2}$.

$$
\operatorname{real}(X(k)), \operatorname{imag}(X(k)) \sim N\left(0,2 W \sigma_{p}^{2}\right) .
$$

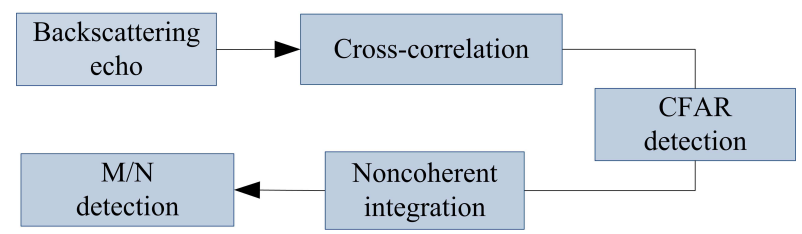

Fig. 2. Scheme of the proposed beam footprint detection method.

According to the probability theory [13], the PDF of $z=|X(k)|^{2}$ is:

$$
P\left(z \mid H_{0}\right)= \begin{cases}\frac{z}{2 W \sigma_{p}^{2}} \exp \left(\frac{z}{2 W \sigma_{p}^{2}}\right) & \text { for } \quad 0 \leq z<\infty, \\ 0 & \text { for } z<0 .\end{cases}
$$

Suppose the number of noncoherent integration is $N_{c}$, then the summation of noise energy is a central chi-square distribution with degrees of $2 N_{c}$ freedom [13]. The PDF is:

$$
P_{x \mid 2 N_{c}, \sigma^{2}}=\frac{x^{N_{c}-1} \exp \left(-\frac{x}{2 W \sigma^{2}}\right)}{\left(2 W \sigma^{2}\right)^{N_{c}} \Gamma\left(N_{c}\right)}, \quad x \geq 0 .
$$

Then the probability of false alarm $P_{\mathrm{f}, \mathrm{cell}}$ is:

$$
P_{\mathrm{f}, \text { cell }}=\int_{V_{2}}^{\infty} \frac{x^{N_{c}-1} \exp \left(-\frac{x}{2 W \sigma^{2}}\right)}{\left(2 W \sigma^{2}\right)^{N_{c}} \Gamma\left(N_{c}\right)} \mathrm{d} x .
$$

For a given false alarm probability $P_{\mathrm{FA}}$, the threshold $V_{2}$ is computed as follows:

(1) The threshold $M_{p}$ is set by the bandwidth of the transmitter's LFM signal.

(2) According to (9) and $M_{p}, P_{\mathrm{f}, \text { cell }}$ can be obtained.

(3) According to (13), we can get the threshold $V_{2}$.

The scheme of the proposed beam footprint detection method is shown in Fig. 2.

\subsection{Beam Footprint Tracking}

In bistatic SAR system, the Doppler bandwidth of the backscattering echo is determined by the relative position between the transmitter's beam footprint and the receiver's beam footprint. Suppose the Doppler centroid and Doppler bandwidth of the bistatic SAR system are known. Based on to the Doppler information of the accumulated echo, the relative position between the transmitter and the receiver can be obtained.

The initial time of the accumulated signal is defined by a certain time when there is no beam footprint overlap between the transmitter and the receiver. During the relative motion of the beam footprint between the transmitter and the receiver, the Doppler spectrum of the accumulated signal is analyzed. To better evaluate the relation between the beam footprint center distance and the Doppler spectrum, the 


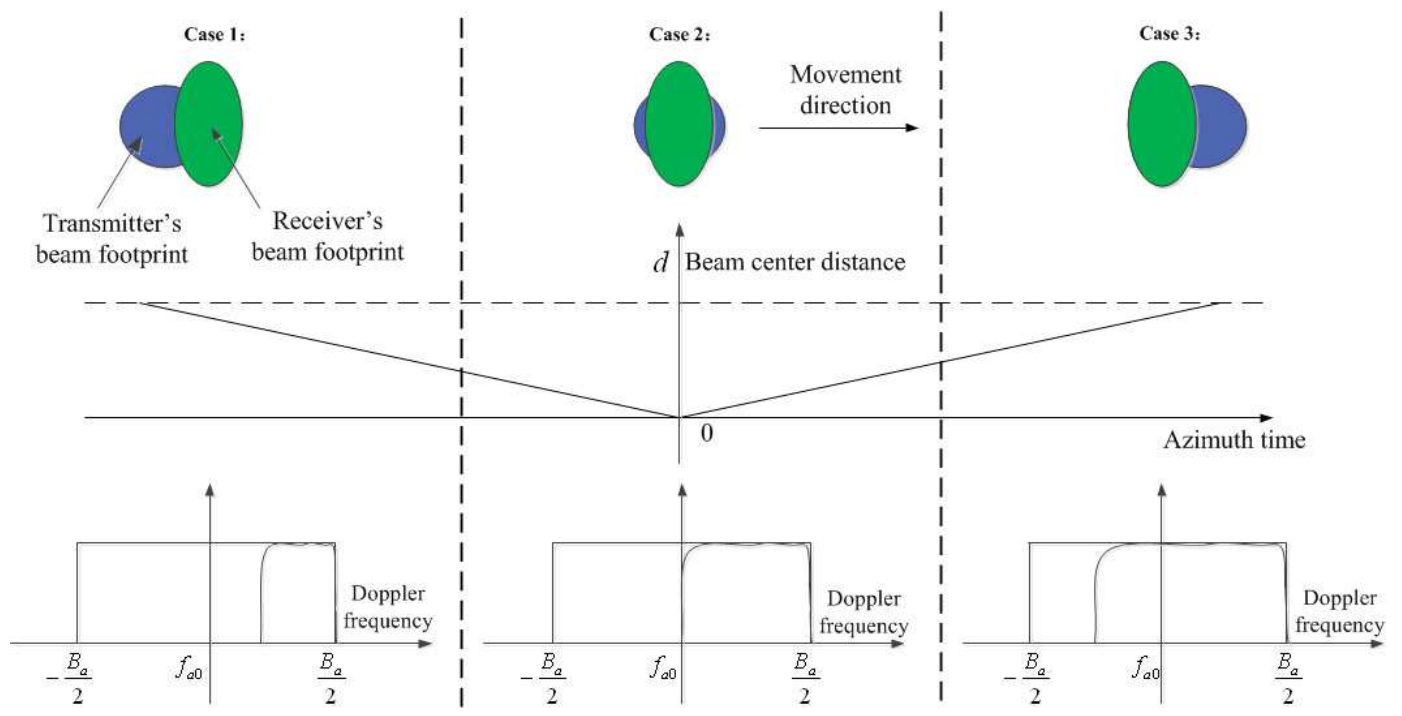

Fig. 3. Doppler spectrum in different relative beam footprint position.

Doppler centroid and Doppler bandwidth are defined by $f_{a 0}$ and $B_{a}$, respectively.

Figure 3 shows the change of the Doppler spectrum during the relative beam footprint motion of the transmitter and the receiver. As can be seen from Fig. 3, with the movement of the transmitter, the beam footprint center distance between the transmitter and the receiver first decreases to zero, and then gradually increase. When the beam footprint center distance is zero, the Doppler bandwidth of the accumulated signal is about $B_{a} / 2$. So the beam footprint center distance can be obtained from the Doppler spectrum of the accumulated signal.

Figure 4 shows the relative beam footprint position of the transmitter and the receiver. $\mathrm{P}$ and $\mathrm{Q}$ are the adjacent PRFs in azimuth time. For a certain target illuminated by the transmitter and the receiver, the Doppler frequency changes linearly with the azimuth time. Suppose the Doppler frequency modulate is $K_{a}$, and the pulse repetition period is $T_{a}$. The initial time of the accumulated signal is defined by a certain time when there is no beam footprint overlap between the transmitter and the receiver. Then the Doppler spectrum of the accumulated signal is analyzed to compute the beam footprint center distance.

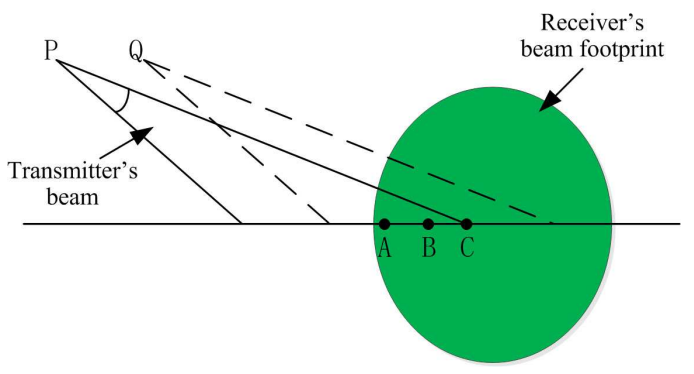

Fig. 4. The relative beam footprint position in the adjacent PRF.
When the transmitter moving to $\mathrm{P}$, we choose some arbitrary targets simultaneous illuminated by the transmitter and the receiver, denoted by Target A, B and C. The Doppler spectrum of the accumulated signal in $\mathrm{P}$ is composed by all targets in the overlap between the transmitter and the receiver. Suppose the Doppler spectrum of the accumulated signal in $\mathrm{P}$ is $\left[f_{\text {ap }}, B_{a} / 2\right]$. When the transmitter moves to $\mathrm{Q}$, due to the decrease of the radial velocity, the instantaneous Doppler frequency of all targets in the overlap between the transmitter and the receiver is reduced by a factor of $K_{a} T_{a}$, and then the low Doppler frequency of the accumulated signal is reduced by a factor of $K_{a} T_{a}$. Consequently, the Doppler spectrum of the accumulated signal in Q is $\left[f_{\text {ap }}-\Delta f, B_{a} / 2\right], \Delta f=K_{a} T_{a}$. During the relative motion of the transmitter and the receiver, the low Doppler frequency is gradually decreased. If the beam footprint center distance is increased to a certain value when the beam overlap is zero or the backscattering echo extended the receiver's range gate, the low Doppler frequency is decreased to $-B_{a} / 2$.

As the Doppler centroid $f_{a 0}$ and Doppler bandwidth $B_{a}$ of the bistatic SAR system are known in advance, we can compute the beam footprint center distance, by using the Doppler information of the accumulated signal received during the relative motion of the transmitter and the receiver. The relation between the beam footprint center distance $d$ and the Doppler spectrum $\left[f_{\mathrm{L}}, f_{\mathrm{H}}\right]$ can be written as:

$$
d=\left\{\begin{array}{r}
\frac{f_{\mathrm{L}}}{K_{a}} \cdot v \text { if } \quad f_{a 0} \leq f_{\mathrm{L}}<f_{a 0}+B_{a} / 2, \\
-\frac{f_{\mathrm{L}}}{K_{a}} \cdot v \text { if } \quad f_{a 0}-B_{a} / 2 \leq f_{\mathrm{L}}<f_{a 0},
\end{array}\right.
$$

where $f_{\mathrm{H}}$ and $f_{\mathrm{L}}$ are the high and low Doppler frequency of the accumulated signal, respectively. 


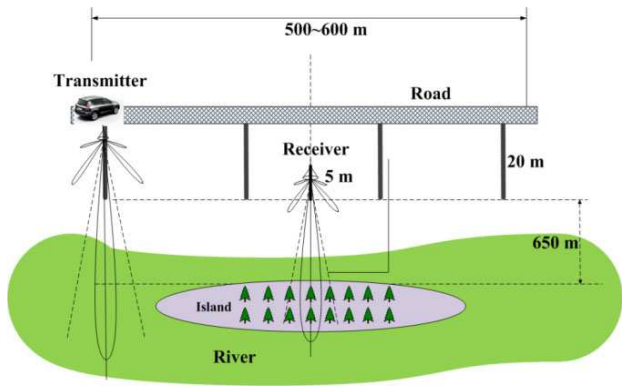

(a)

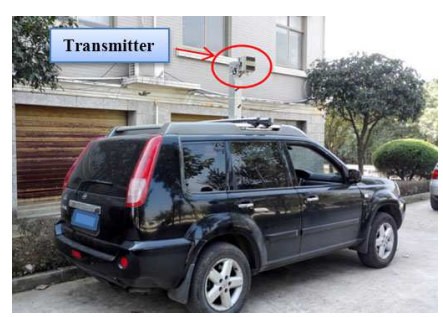

(b)

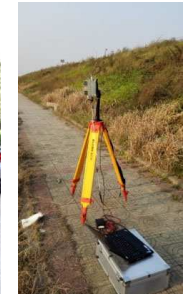

(c)
Fig. 5. Geometry of vehicle-based bistatic SAR experiment. (a) Sketch map of the experiment; (b) Vehicle-based transmitter; (c) Receiver.

If the beam footprint center distance satisfies the predefined condition, the receiver's beam footprint is moving to realize the the transmitter's beam footprint tracking.

However, as the energy of the backscattering echo has nonlinear variation over the azimuth time in an inhomogeneous observational area, the estimate error of the Doppler frequency spectrum is increased and the beam tracking performance becomes poorer. So the proposed beam footprint tracking method has better performance in homogeneous area.

\section{Bistatic SAR Experiments}

\subsection{Vehicle-based Bistatic SAR Experiment}

The bistatic SAR experiment which uses noncooperative spaceborne radar as illuminating of opportunity is too complex to perform. In order to evaluate the performance of the proposed beam footprint detection approach, a vehicle-based bistatic SAR experiment with a Ku-band mini SAR system was carried out along the elevated highway in the south of Changsha, China.

The vehicle-based Ku-band mini SAR system is used as the non-cooperative transmitter and the receiver is a fixed $\mathrm{Ku}$-band mini SAR system in the ground to receive the direct signal and the backscattering echo. As the elevation angle of the receiver is almost zero, the backscattering echo is very weak.

Figure 5(a) shows the geometry of the vehicle-based bistatic SAR experiment. The experimental SAR system is operating at Ku-band with Frequency-Modulated Continuous Wave (FMCW). The Ku-band mini SAR system is mounted

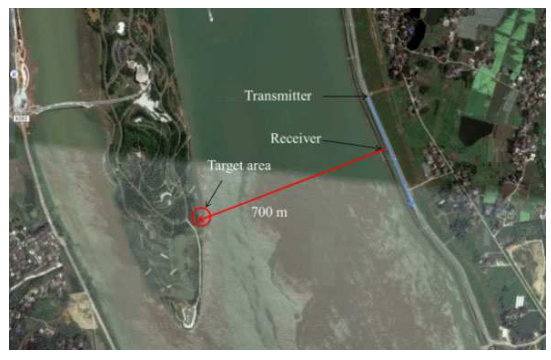

(a)

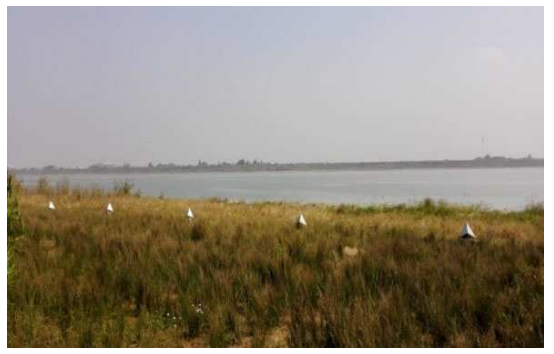

(b)

Fig. 6. The observational scene. (a) Google map of the scene; (b) point targets.

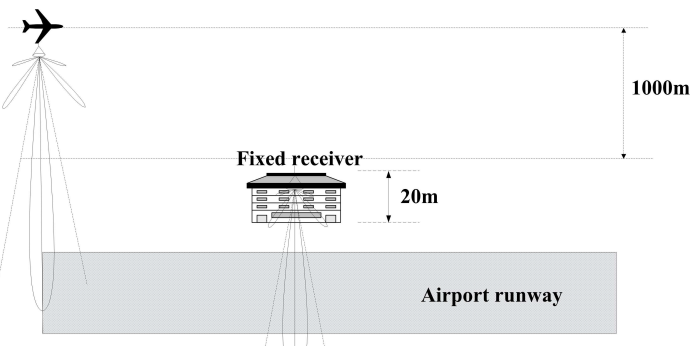

$\wedge \wedge \wedge \wedge \wedge \wedge \wedge \wedge \wedge \wedge \wedge$

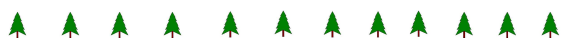

(a)

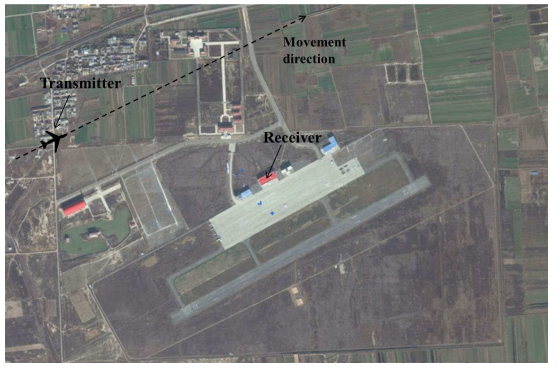

(b)

Fig. 7. Airborne bistatic SAR experiment. (a) Scheme of the experiment; (b) Google map of the scene.

on the roof of a sport utility vehicle (SUV), which is shown in Fig. 5(b). The vehicle-based transmitter was used as a non-cooperative transmitter which moves along a straight elevated road. The height of the elevated road is about $20 \mathrm{~m}$ higher than the observational area. The velocity of the car is about $16 \mathrm{~m} / \mathrm{s}$. The stationary receiver was fixed in the ground which is about $5 \mathrm{~m}$ higher than the observational scene.

The observational scene is an island in the river, which is shown in Fig. 6(a). During the vehicle-based bistatic SAR 
experiment, some corner reflectors are placed in the observational scene, which is shown in Fig. 6(b).

\subsection{Airborne Bistatic SAR Experiment}

In bistatic SAR system, the beam footprint center distance between the transmitter and the receiver can be obtained according to the Doppler spectrum of the accumulated signal. In this section, the proposed beam footprint tracking method is validated by airborne bistatic SAR experiment.

Figure 7(a) shows the sketch map of the airborne bistatic SAR experiment. The google map of the scene is shown in Fig. 7(b). The transmitter is mounted in an airplane, and the receiver is fixed on the roof of a building. The transmitter and the receiver are all working in vertical stripmap mode. The angle of the antenna is $60^{\circ}$.

On the one hand, based on the GPS equipment in the airborne transmitter and the fixe-receiver, the beam footprint center distance between the transmitter and the receiver can be obtained in any azimuth time. As the Doppler centroid and Doppler bandwidth of the airborne bistatic SAR system are known in advance, the ideal Doppler frequency spectrum of the accumulated signal can be acquired in any azimuth time. On the other hand, the beam footprint center distance can be acquired from the Doppler frequency of the accumulated backscattering signal. Consequently, the validation and performance of the proposed beam footprint tracking method can be obtained by comparing the beam footprint center distance computed from the Doppler spectrum and the GPS equipment.

\section{Experiment Results}

\subsection{Beam Footprint Detection}

Two different experiments are performed to evaluate the validation and performance of the proposed beam footprint detection method. In the first experiment, the transmitter and the receiver are all working in vertical stripmap mode. In the second experiment, the transmitter is working in squint stripmap mode, and the receiver is working in vertical stripmap mode. The results of the two experiments are shown below.

\section{(1) The transmitter and the receiver are all working in vertical stripmap mode}

Figure 8(a) shows the amplitude of the adjacent sampling signals. Fig. 8(b) shows the cross-correlation results of the adjacent sampling signals and adjacent sampling noises. As the adjacent backscattering echoes have high correlation and the adjacent noises are independent, it is clearly seen that there is a peak value in the middle of the backscattering echo's cross-correlation result. The false alarm probability of CFAR detection is set at $10^{5}$. Based on the sampling noise, the Monte-Carlo simulations are carried out to compute the threshold $V_{T}$ (The red line in Fig. 8(b)).
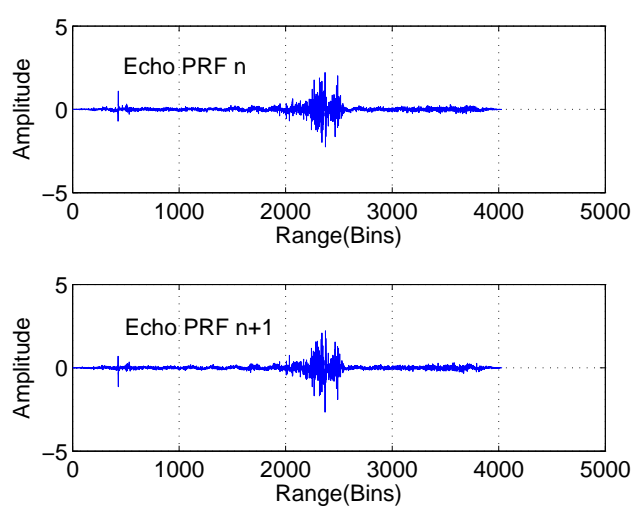

(a)
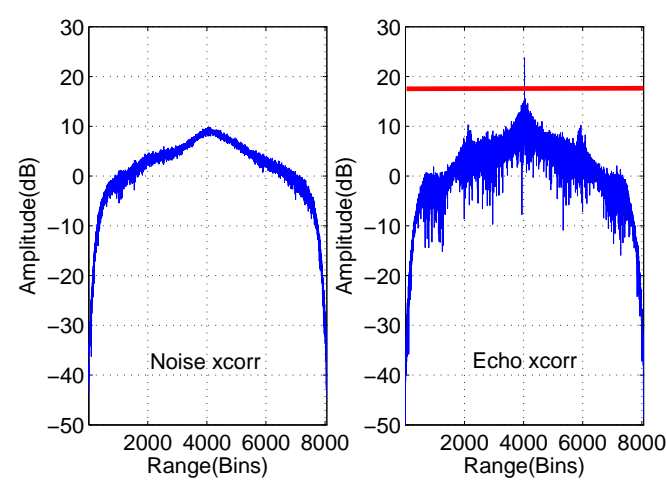

(b)
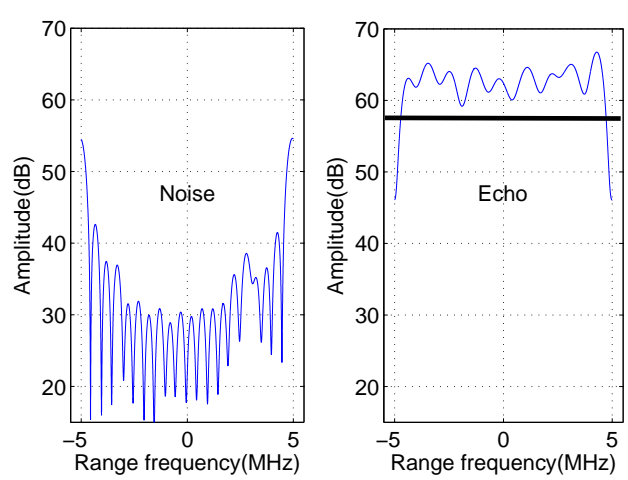

(c)

Fig. 8. Experimental results. (a) Experimental echoes of adjacent PRFs; (b) Cross-correlation results; (c) FFT of cross-correlation results.

In the $\mathrm{M} / \mathrm{N}$ detection processing, $\mathrm{M}$ is the sampling point number in the range direction. Since the range bandwidth $B_{r}$ of the backscattering echo is known in advance, so $\mathrm{N}$ is set at INT $\left(B_{r} \cdot F_{S}\right)$, where $F_{S}$ is the sampling frequency. The false alarm probability of $\mathrm{M} / \mathrm{N}$ detection is also set at $10^{5}$, then according to (9) and $\mathrm{N}$, the threshold $V_{2}$ (The black line in Fig. 8(c)) can be obtained. The range bandwidth of the Ku-band mini SAR system after dechirp processing is $8 \mathrm{MHz}$. As can be seen from Fig. 8(c), the range bandwidth of the backscattering echo's cross-correlation result agrees well with $8 \mathrm{MHz}$. 


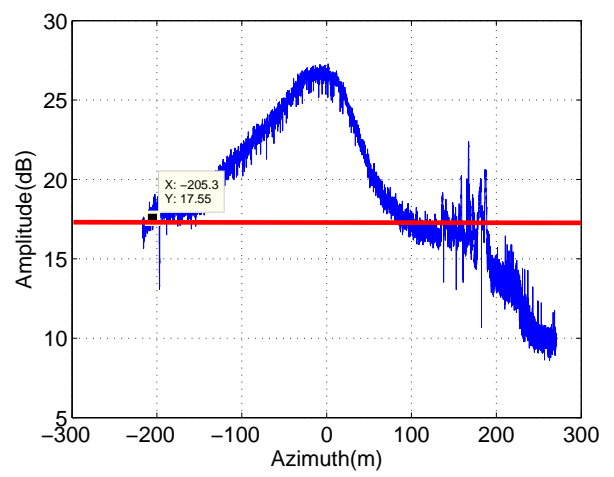

(a)

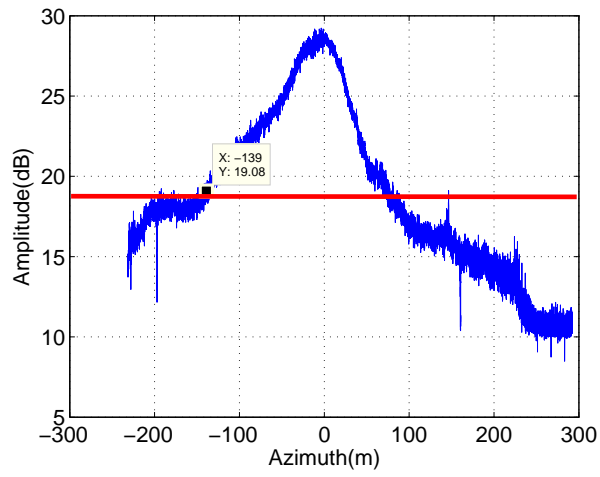

(b)

Fig. 9. Cross-correlation results of the echoes. (a) Pass 1; (b) Pass 2.

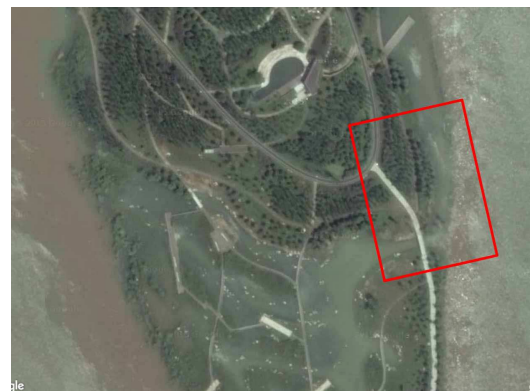

(a)

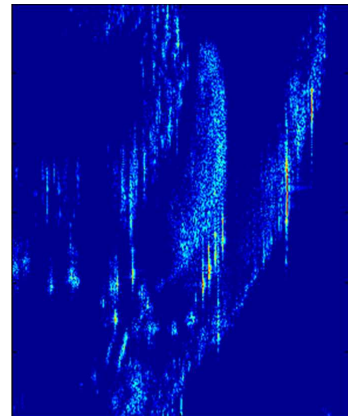

(b)

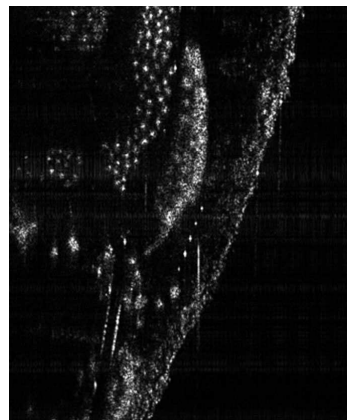

(c)
Fig. 10. Image of the observational scene. (a) Observational area; (b) Bistatic image; (c) Monostatic imag.

According to $V_{T}$ and $V_{2}$, the performance of the beam footprint detection method is evaluated by the experimental

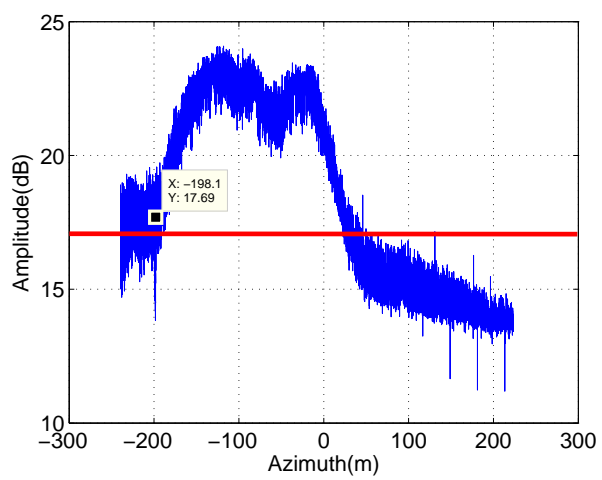

Fig. 11. Cross-correlation result.

data. Fig. 9 shows the maximum values of the adjacent echo's cross-correlation results and the threshold $V_{T}$. The marker in Fig. 9 shows the position where the detection method can realize stable detection of the transmitter's beam footprint.

The width of the receiver's beam footprint in the azimuth direction is about $90 \mathrm{~m}$. As can be seen from Fig. 9(a) and 9(b), the proposed method can even realize beam footprint detection in the sidelobe of the receiver, so the transmitter's beam footprint can be detected in advance. Then the Doppler frequency spectrum of the sampling signal is analyzed to compute the beam footprint distance.

The observational area is an island in the river which is shown in Fig. 10(a). As there is no synchronization link between the transmitter and the receiver, the conventional motion error correction method cannot be used to realize accurate imaging of the scene [16].

Figure 10(a) shows the bistatic image without synchronization process [17]. The monostatic image with motion error correction approach is shown in Fig. 10(b). From Fig. 10, we can clearly see that the bistatic SAR image fit very well with the observational scene and the monostatic image. The resolution of the bistatic SAR image is about $0.85 \mathrm{~m}$ (azimuth) $\times 0.35 \mathrm{~m}$ (range). The ideal bistatic resolution is $0.6 \mathrm{~m}$ (azimuth) $\times 0.3 \mathrm{~m}$ (range). As the transmitter is non-cooperative, then the motion compensation technique cannot perform. So the resolution of the bistatic SAR image is poorer than the ideal value. Still, the bistatic SAR image and the monostatic SAR image are almost the same, which validate that we can get the coarse image of the observational scene in non-cooperative bistatic SAR system.

\section{(2) The transmitter is working in squint stripmap mode,} and the receiver is working in vertical stripmap mode

In this experiment, the transmitter is working in squint stripmap mode, and the squint angle is about $8^{\circ}$. The receiver is working in vertical stripmap mode. The cross-correlation result of the sampling signal is shown in Fig. 11.

As the transmitter is working in squint stripmap mode, so the peak value is deviate from the center position of the cross-correlation result. According to the squint angle and the slant range, the ideal position of the peak value is about $-115 \mathrm{~m}$. As can be seen from Fig. 11, the peak value position 


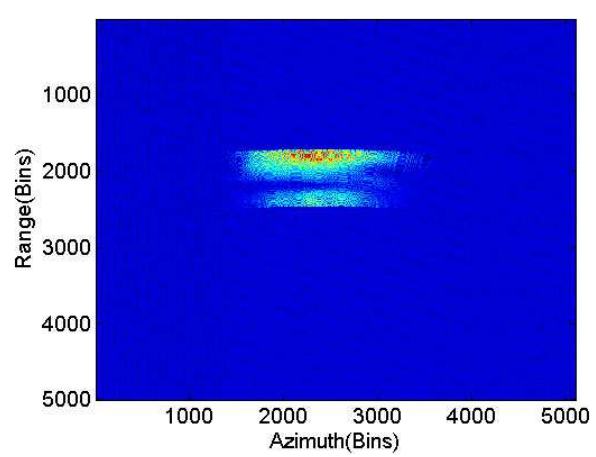

(a)

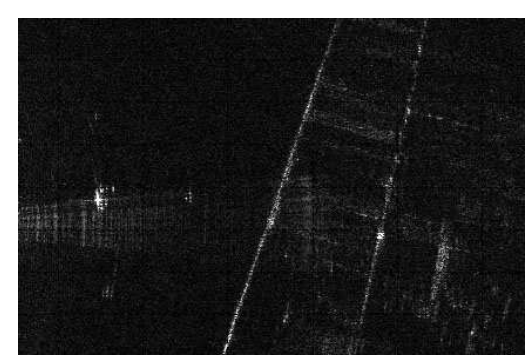

(b)

Fig. 12. Amplitude of the backscattering echo and bistatic image. (a) Amplitude of the backscattering echo; (b) Image of the scene.

of the cross-correlation is about $-120 \mathrm{~m}$, which agrees well with the ideal position. As can be seen from Fig. 11, the proposed method can also realize beam footprint detection in the sidelobe of the receiver in squint stripmap mode.

Due to the limitation of vehicle-based experimental conditions, the elevation angle of the transmitter and the receiver are all very small, so the backscattering echo is very weak. However, the proposed beam footprint detection method is validated by the cross-correlation result of the vehicle-based experiment. Experiment results show that the proposed method can realize stable beam footprint detection in the sidelobe of the receiver.

\subsection{Beam Footprint Tracking}

Due to the limitation of vehicle-based experimental conditions, the proposed beam footprint tracking method is validated by airborne bistatic SAR experiment in this section. The transmitter and the receiver are all working in vertical stripmap mode.

Figure 12(a) and 12(b) show the amplitude of the backscattering echo and image of the scene, respectively. As can be seen from Fig. 12, the energy of the echo is mainly distributed in the overlap area of the beam footprint. On both sides of the echo, the sampling signal is mainly noise, because the transmitter and the receiver have little overlap.

Figure 7(a) shows the relative position of the transmitter and the receiver. According to the GPS information between the transmitter and the receiver, the real beam footprint center distance is shown in Fig. 13(a). Suppose the initial

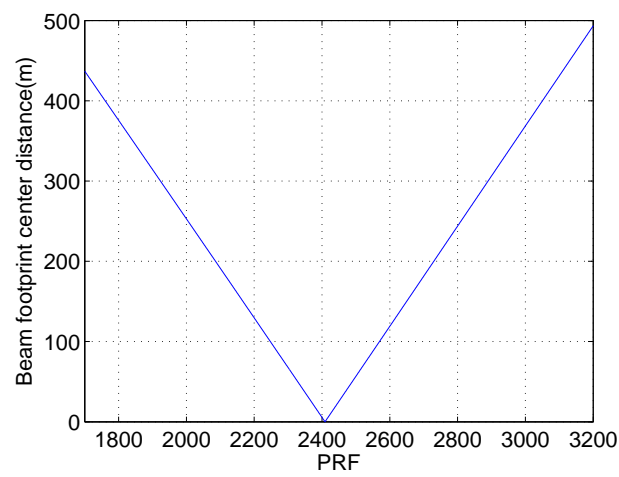

(a)

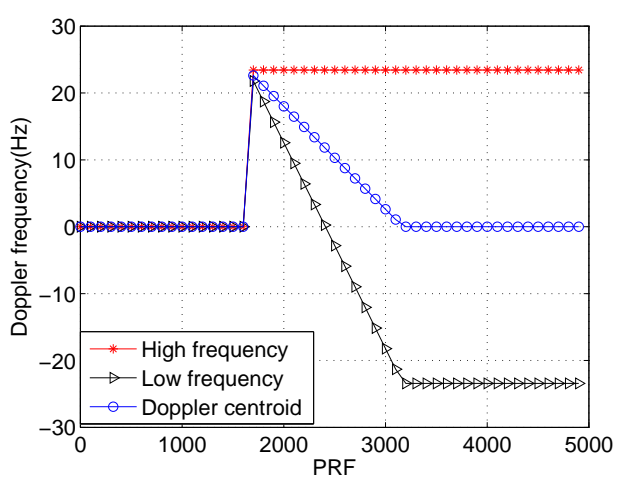

(b)

Fig. 13. Beam footprint center distance and Doppler frequency information. (a) Beam footprint center distance from GPS; (b) Ideal Doppler frequency.

accumulation position is 1 . Then from (14), we can derive the ideal Doppler frequency information of the airborne bistatic SAR system which is shown in Fig. 13(b). Because the initial accumulation position is fixed, so the high Doppler frequency is stable with the increasing beam footprint overlap. The low Doppler frequency is gradually decreased. When the beam footprint center distance between the transmitter and the receiver is zero, the low Doppler frequency is about 0. If the beam footprint center distance is increased to a certain value when the beam overlap is zero or the backscattering echo extended the receiver's range gate, the low Doppler frequency is decreased to $-B_{a} / 2$.

Figure 14(a) and 14(b) shows the Doppler frequency information of the airborne bistatic SAR experiment. Because the initial accumulation position is fixed, the high Doppler frequency of the experiment is stable with the increasing beam footprint overlap. The low Doppler frequency is gradually decreased. Comparing Fig. 13(b) and Fig. 14(b), it can be found that the experiment result fit very well with the ideal result. The estimated beam footprint center distance is shown in Fig. 14(c).

The beam footprint tracking error of the proposed method is shown in Tab. 1. The error is indicated by the deviation of the estimated footprint center distance with respect to the real distance from the GPS information. The azimuth width of the receiver's beam footprint is about $900 \mathrm{~m}$. 


\begin{tabular}{|c|c|c|c|c|c|}
\hline $\begin{array}{c}\text { Accumulated } \\
\text { PRF }\end{array}$ & $\begin{array}{c}\text { Low Doppler } \\
\text { frequency (Hz) }\end{array}$ & $\begin{array}{c}\text { Doppler } \\
\text { centroid (Hz) }\end{array}$ & $\begin{array}{c}\text { Beam footprint center } \\
\text { distance from Doppler (m) }\end{array}$ & $\begin{array}{c}\text { Beam footprint center } \\
\text { distance from GPS (m) }\end{array}$ & $\begin{array}{c}\text { Beam tracking } \\
\text { error (m) }\end{array}$ \\
\hline $1-1700$ & 20.9 & 19.95 & 402.69 & 436.98 & -34.29 \\
\hline $1-1800$ & 18.6 & 19.13 & 358.38 & 375.76 & -17.38 \\
\hline $1-2000$ & 13.1 & 16.17 & 252.4 & 252.96 & -0.56 \\
\hline $1-2200$ & 6.8 & 12.83 & 131.02 & 129.55 & 1.47 \\
\hline $1-2409$ & -1.1 & 10.75 & 21.19 & 0 & 21.19 \\
\hline $1-2500$ & -4.4 & 7.95 & 84.77 & 56.65 & 28.12 \\
\hline $1-3000$ & -17.9 & 0.95 & 344.89 & 386.61 & -41.72 \\
\hline $1-3200$ & -23.2 & -0.55 & 447.01 & 493.36 & -46.35 \\
\hline
\end{tabular}

Tab. 1. Beam footprint tracking errors.

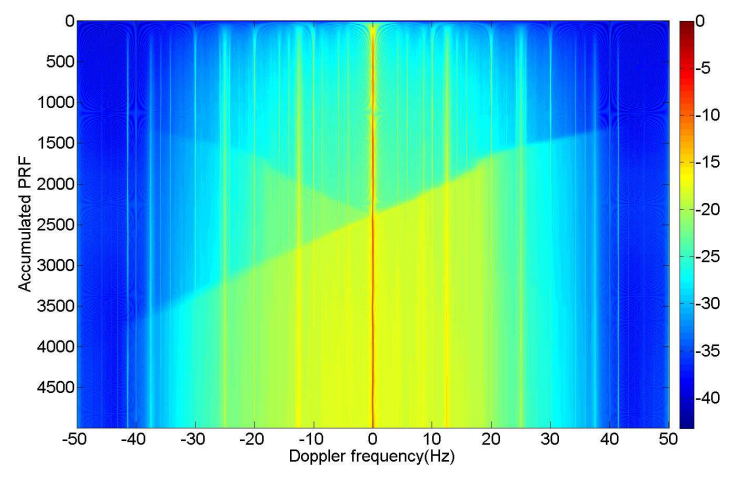

(a)

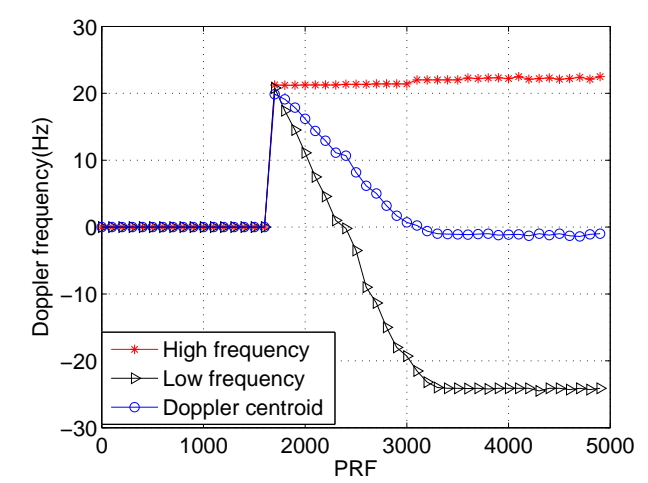

(b)

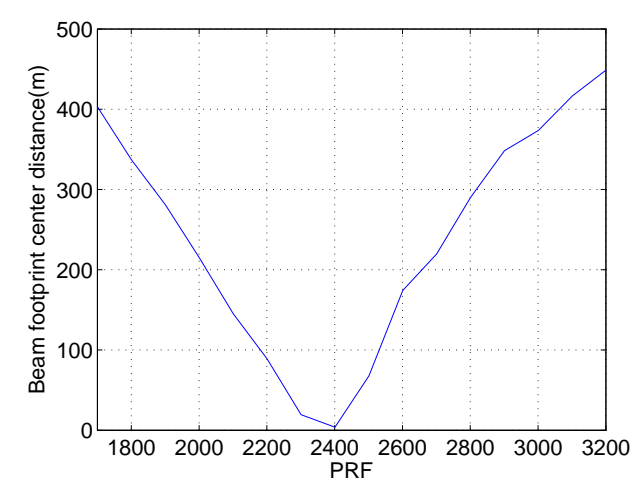

(c)

Fig. 14. Airborne bistatic SAR experiment result. (a) Doppler frequency spectrum changes with the accumulated signal; (b) Doppler information of the accumulated signal; (c) Beam footprint center distance from Doppler spectrum.
Table 1 shows the beam footprint tracking errors of the proposed beam footprint tracking method. As can be seen from Tab. 1, due to the sidelobe of the receiver's beam footprint in the initial and end positions, the Doppler frequency errors are larger. The maximum beam footprint center distance error equals to $-46.35 \mathrm{~m}$, which is about $5 \%$ of the receiver's beam footprint width. Meanwhile, the Doppler centroid of the backscattering echo is decreased from $B_{a} / 2$ to zero with the movement of the transmitter's beam footprint.

Due to the limitation of the airborne bistatic SAR experiment, the observational area is mainly low scattering coefficient area, so the Doppler frequency errors are larger. Although the maximum beam footprint tracking error is about $5 \%$ of the receiver's beam footprint width, the performance of the proposed beam footprint tracking method can be well validated by the airborne bistatic SAR experiment result.

\section{Conclusion}

In this paper, an efficient transmitter beam detection and tracking method for non-cooperative bistatic SAR is presented. Based on cross-correlation and Doppler frequency information of the backscattering echo, the efficiency of scene raw data detection and tracking is greatly improved. The results of the vehicle-based non-cooperative bistatic SAR experiment show that the proposed detection method can realize transmitter beam footprint detection in the sidelobe of the receiver. Furthermore, the results of airborne bistatic SAR experiment show that the proposed tracking method can well realize transmitter's beam footprint tracking in non-cooperative bistatic SAR. On the basis of the proposed beam footprint detection and tracking method in this paper, non-cooperative spaceborne/airborne bistatic SAR experiment and real-time processing will be topics for future research.

\section{Acknowledgments}

This work was partly supported by National Natural Science Foundation of China (Grant Nos. 61501477), the research project of National University of Defense Technology (Grant No. CJ12-04-02). 


\section{References}

[1] YANG, Y. H., PI, Y. M., LI, R. Considerations for non-cooperative bistatic SAR with spaceborne radar illuminating. In CIE International Conference on Radar. Shanghai (China), 2006, p. 1-4. DOI: 10.1109/ICR.2006.343395

[2] TIAN, W. M., LONG, T., YANG, J., et al. General processing approach for bistatic SAR systems: description and performance analysis. In 8th European Conference on Synthetic Aperture Radar (EU$S A R)$. Aachen (Germany), 2010, p. 1-4.

[3] YAN, F. F., CHANG, W. G., LI, X. Y. Beam-footprint detection for non-cooperative spaceborne/airborne bistatic SAR. In Progress in Electromagnetics Research Symposium. Prague (Czech Republic), 2015, p. 604-608.

[4] GE, X. J., HE, Y., SONG, J. Cross-correlation detection and time difference estimation in non-cooperative bistatic radar systems. In IEEE International Conference on Signal Processing. Beijing (China), 2010, p. 2261-2265. DOI: 10.1109/ICOSP.2010.5655147

[5] ANTONIOU, M., CHERNIAKOV, M. GNSS-based bistatic SAR: a signal processing view. EURASIP Journal on Advances in Signal Processing, 2013, vol. 2013, no. 98, p. 124-132. ISSN: 1687-6180. DOI: $10.1186 / 1687-6180-2013-98$

[6] WANG, H. Y., WU, Y. H. The accurate model for beam synchronization in spaceborne/airborne hybrid bistatic SAR. In CIE International Conference on Radar. Chengdu (China), 2011, p. 950-953. DOI: 10.1109/CIE-Radar.2011.6159698

[7] WANG, W. Q., CAI, J. Y. Antenna directing synchronization for bistatic synthetic aperture radar systems. IEEE Antennas Wireless Propagation Letters, 2010, vol. 9, no. 2, p. 307-310. ISSN: 15361225. DOI: 10.1109/LAWP.2010.2047490

[8] MU, F. Y., ZHANG, J. F., DU, J. A weak signal detection technology based on stochasticresonance system. In International Conference on Computer Science and Service System, Nanjing (China), 2011, p. 2004-2007. DOI: 10.1109/CSSS.2011.5974613

[9] YU, D., WU, J. Z., CHEN, Z. P. Detection of LFM signals in low SNR based on STFT and wavelet denoising. In International Conference on Audio Language and Image Processing. Shanghai (China), 2014, p. 921-925. DOI: 10.1109/ICALIP.2014.7009929

[10] WALTERSCHEID, I., EDPETER, T., ENDER, J. H. Performance analysis of a hybrid bistatic SAR system operating in the double sliding spotlight mode. In IEEE Transactions on Geoscience and Remote Sensing Symposium. Barcelona (Spain), 2007, p. 2144-2147. DOI: $10.1109 /$ IGARSS.2007.4423258

[11] ZHOU, P., PI, Y. M. Two methods for beam synchronization in spaceborne/airborne hybrid bistatic SAR. Acta Electronica Sinica, 2009, vol. 37, no. 6, p. 1192-1197. ISSN: 0372-2112. DOI: $10.3321 /$ j.issn:0372-2112.2009.06.008

[12] NICO, G., TESAURO, M. On the existence of coverage and integration time regimes in bistatic SAR configurations. IEEE Geoscience and Remote Sensing Letters, 2007, vol. 4, no. 3, p. 426-430. ISSN: 1545-598X. DOI: 10.1109/LGRS.2007.895957

[13] ANINDYA, D. Beyond the central limit theorem: asymptotic expansions and pseudorandomness for combinatorial sums. In IEEE 56th Annual Symposium on Foundations of Computer Science (FOCS). Berkeley (USA), 2015, p. 883-902. DOI: 10.1109/FOCS.2015.59
[14] YANG, X. L., WEN, G. J., MA, C. H., et al. CFAR detection of moving range-spread target in white Gaussian noise using waveform contrast. IEEE Geoscience and Remote Sensing Letters, 2016, vol. 13, no. 2, p. 282-286. ISSN: 1545-598X. DOI: $10.1109 /$ LGRS.2015.2511060

[15] LUHR, D., ADAMS, M. Radar noise reduction based on binary integration. IEEE Sensors Journal, 2014, vol. 15, no. 3, p. 766-777. ISSN: 1530-437X. DOI: 10.1109/JSEN.2014.2352295

[16] PU, W., LI, W. C., LV, Y. X., et al. An extended omega-K algorithm with integrated motion compensation for bistatic SAR. In IEEE Radar Conference. Arlington (USA), 2015, p. 1291-1295. DOI: 10.1109/RADAR.2015.7131194

[17] NEO, Y. L., WONG, F. H., CUMMING, I. G. Processing of azimuthinvariant bistatic SAR data using the range Doppler algorithm. IEEE Transactions on Geoscience and Remote Sensing, 2008, vol. 46, no. 1 p. 14-21. ISSN: 0196-2892. DOI: 10.1109/TGRS.2007.909090

\section{About the Authors ...}

Feifei YAN was born in Henan, P.R.China in 1987. He received the B.S. degree in Electronic Information Engineering from Xidian University, Xian, China, in 2005, and the M.S. degree in Information Engineering from the National University of Defense Technology, Changsha, China, in 2011. $\mathrm{He}$ is currently working towards the $\mathrm{Ph}$. D. degree in Information Engineering at the National University of Defense Technology. His current research interests include bistatic radar systems, and parameter estimation.

Wenge CHANG was born in Shannxi, P.R.China in 1965. He received the M.S. and Ph.D. degrees in Information and Communication Engineering from the National University of Defense Technology, Changsha, China, in 1993 and 2001, respectively. He is currently a Professor with the National University of Defense Technology. His fields of interest include synthetic aperture radar systems and signal processing.

Xiangyang LI was born in Hunan, P.R.China in 1972. He received the M.S. and Ph.D. degrees in Information and Communication Engineering from the National University of Defense Technology, Changsha, China, in 1996 and 2000, respectively. He is currently a Professor with the National University of Defense Technology. His fields of interest include synthetic aperture radar systems and signal processing.

Qilei ZHANG was born in Gansu, P.R.China in 1985. He received the M.S. and Ph.D. degrees in Information and Communication Engineering from the National University of Defense Technology, Changsha, China, in 2009 and 2014, respectively. He is currently a Lecturer with the National University of Defense Technology. His current research interests include bistatic radar systems, and signal processing. 